\title{
ENERGY CONVERSIONS AND STORAGE CAUSED BY AN UNSTEADY POLOIDAL FLOW IN ACTIVE SOLAR REGIONS
}

\author{
ZHONGYUAN LI \\ Dr Remeis-Sternwarte, Astronomical Institute, Erlangen-Nürnberg University, Bamberg, F.R.G. \\ and \\ Dept. of Earth and Space Science, University of Science and Technology of China, Hefei, Anhui, China \\ and \\ W. R. HU \\ Institute of Mechanics, Academia Sinica, Beijing, China
}

(Received 21 January, 1988)

\begin{abstract}
In this paper we discuss coupling processes between a magnetic field and an unsteady plasma motion, and analyze the features of energy storage and conversions in active region.

It is pointed out that the static force-free field is insufficient for a discussion of storage processes, and also the pure unsteady plasma rotation is not a perfect approach. In order to analyze the energy storage, we must consider the addition of poloidal plasma motion. The paper shows that because the unsteady poloidal flow is added and coupling occurs between the magnetic field and both the toroidal and the poloidal plasma flows, an unsteady process is maintained which changes the force-free factor with time. Hence, the energy in the lower levels can be transferred to the upper levels, and a considerable energy can be stored in the active region. Finally, another storage process is given which is due to the pure poloidal flow. The article shows that even if there is no twisted magnetic line of force, the energy in the lower levels may still be transferred to the upper levels and stored there.
\end{abstract}

\section{Basic Equations}

The force-free field has extensive applications in the solar and space physical environment. The magnetic configuration is the total force-free field in the active region. Generally, we should construct theoretical models of the force-free fields first, and then, compare them with the observations. The force-fre condition is

$$
(1 / c)(\mathbf{j} \times \mathbf{B})=0,
$$

namely,

$$
\nabla \times \mathbf{B}=\alpha(t, \mathbf{r}) \mathbf{B},
$$

where the force-free factor $\alpha(t, \mathbf{r})$ depends on both, the time $t$ and the place coordinates $\mathbf{r}$. There is an assorted plasma motion in the active region. The induction equation of the magnetic field is as

$$
\partial \mathbf{B} / \partial t=\nabla \times(\mathbf{V} \times \mathbf{B})+\eta \nabla^{2} \mathbf{B} .
$$

Because the magnetic Reynold number $R_{m}$ is usually much larger than 1 in the solar atmosphere, that is 


$$
R_{m}=U L / \eta_{m} \cong 10^{3} \gg 1,
$$

the magnetic field will satisfy the frozen condition, and Equation (1.3) may be reduced to

$$
\partial \mathbf{B} / \partial t=\nabla \times(\mathbf{V} \times \mathbf{B}) .
$$

The condition of zero divergence of the magnetic field is

$$
\nabla \cdot \mathbf{B}=0 .
$$

Now we establish a cylindrical-coordinate system for the axial-symmetric force-free magnetic field. According to $\nabla \cdot \mathbf{B}=0$, we introduce the magnetic potential function $A$ as

$$
B_{r}=(1 / r)(\partial A / \partial z), \quad B_{z}=(-1 / r)(\partial A / \partial r)
$$

Substituting Equation (1.6) into the force-free condition (1.2), we have

$$
\begin{aligned}
& r B_{\theta}=G(A, t), \quad \alpha=-\partial G(A, t) / \partial A, \\
& \Lambda(A)=-G(A, t)[\partial G(A, t) / \partial A]
\end{aligned}
$$

where $\Lambda$ is a differential operator

$$
\Lambda=\left(\frac{\partial^{2}}{\partial r^{2}}-\frac{1}{r} \frac{\partial}{\partial r}+\frac{\partial^{2}}{\partial z^{2}}\right) .
$$

Substituting Equations (1.7) and (1.8) into Equation (1.4), we obtain the component forms of Equation (1.4), as

$$
\begin{aligned}
& \partial A / \partial t+u(\partial A / \partial r)+w(\partial A / \partial z)=0 \\
& \begin{aligned}
\partial G(A, t) / \partial t+(\partial u / \partial r & -u / r+\partial w / \partial z) G(A, t)= \\
& =-(\partial v / \partial z)(\partial A / \partial r)+(\partial v / \partial r-v / r)(\partial A / \partial 2) .
\end{aligned}
\end{aligned}
$$

According to the kinematical momentum conservation, the following constraint is easily obtained

$$
\mathbf{B} \cdot[\partial \mathbf{V} / \partial t+(\mathbf{V} \cdot \nabla) \mathbf{V}+\nabla p / \rho]=0
$$

where $\mathrm{V}=(u, v, w)$ is the velocity field in cylindrical coordinates.

Under the kinematical condition, the force-free magnetic field can be determined as long as we know the distribution features of the force-free factor. Of course, when studying the evolution of the kinematical force-free field, the influence of a reasonable flow on the variations of the magnetic field should be taken into account, independent on whether the processes might be described by the solutions of Equations (1.6)-(1.11) or not. 


\section{The Unsteady Plasma Rotation}

In the 1960's, Gold and Hoyle (1960) suggested that the magnetic lines of force may be twisted in the solar active region. A few authors, Barnes and Sturrock (1972), Ma (1981), and Švestka (1977) have subsequently calculated the quantitative relationship between the static force-free field connecting the magnetic field and the twisting processes. They pointed out that the potential magnetic field without the current may be twisted into the force-free field with the enhanced current produced by the plasma rotation. They thought that it might be due to the storage energy in the active region. Lately, Li and $\mathrm{Hu}$ (1982a, b, 1984) have stated that the processes should be unsteady, and especially that they should not be static.

Let us go a step further and discuss this problem now. First, we analyze the evolution of the magnetic field configuration due to the change of angular velocity $\Omega$ with time $t$. With pure plasma rotation, the distribution of the flow field is

$$
u=0, \quad v=v(r, z, t), \quad w=0 .
$$

Substituting Equation (2.1) into (1.9), we have

$$
\partial A / \partial t=0
$$

Differentiating above formula with $r$ and $z$, respectively, and applying relationship (1.6), we get

$$
\partial B_{r} / \partial t=0, \quad \partial B_{z} / \partial t=0
$$

Using Ampère's law, we immediately obtain

$$
\frac{\partial j_{\theta}}{\partial t}=\frac{\partial}{\partial t}\left[\frac{c}{4 \pi}\left(\frac{\partial B_{r}}{\partial z}-\frac{\partial B_{z}}{\partial r}\right)\right]=0 .
$$

Obviously, both the poloidal magnetic field and the toroidal current cannot be changed by the plasma rotation whether the angular velocity $\Omega$ is a function of time $t$ or not.

We will now discuss the change of the toroidal magnetic field. Substituting the velocity field (2.1) into (1.10), we get

$$
\partial G(A, t) / \partial t=-r[(\partial \Omega / \partial z)(\partial A / \partial r)-(\partial \Omega / \partial r)(\partial A / \partial z)]
$$

Since $\Lambda(A)$ is not a function of time $t$ in Equation (1.8), we have

$$
\partial^{2} G^{2}(A, t) / \partial A \partial t=0
$$

Hence, we get

$$
G^{2}(A, t)=f^{2}(t)+G_{1}^{2}(A) .
$$

Substituting Equation (2.7) into (1.7), we obtain

$$
\begin{aligned}
& B_{\theta}(r, z, t)=(1 / r)\left[f^{2}(t)+G_{1}^{2}(A)\right]^{1 / 2}, \\
& \alpha(r, z, t)=-G_{1}(A) G_{1}^{\prime}(A)\left[f^{2}(t)+G_{1}^{2}(A)\right]^{-1 / 2} .
\end{aligned}
$$


When substituting Equations (2.8) and (2.9) into the force-free equation, Equation (1.8) becomes

$$
\Lambda(A)=\left[-G_{1}(A)\right]\left[\mathrm{d} G_{1}(A) / \mathrm{d} A\right] .
$$

Now we can introduce the steady relationships as

$$
\alpha_{i}=-\mathrm{d} G_{1}(A) / \mathrm{d} A, \quad r B_{\theta 1}=G_{1}(A) .
$$

We find that the total toroidal magnetic field given by Equation (2.8) is stronger than the steady one given by (2.11). The absolute value of the total force-free factor defined by (2.9) is less than that of the steady one, namely $B_{\theta}>B_{\theta 1},|\alpha|<\left|\alpha_{i}\right|$. The results show that after the magnetic flux tube is twisted by the unsteady plasma rotation, the magnetic field is relatively stronger, while the gradient is relatively smaller. If $\alpha_{i}$ is given, the poloidal field may be completely determined for the boundary value problem described by Equation (2.10); the change of the toroidal field with time is then given by Equation (2.8).

Substituting Equation (2.7) into (2.5), we get the connection between the toroidal field and the angular velocity

$$
f(t) f^{\prime}(t) /\left[f^{2}(t)+G_{1}^{2}(A)\right]^{1 / 2}=-(r \partial A / \partial r)(\partial \Omega / \partial z)+(r \partial A / \partial z)(\partial \Omega / \partial r) .
$$

If $G_{1}(A)$ is given, the magnetic potential $A(r, z)$ can be derived from Equation (2.10). Then integrating (2.12) with time $t$, we can reduce the resultant equation to

$$
\begin{aligned}
f^{2}(t)= & f^{2}(0)+\left[f^{2}(0)+G^{2}(A)\right]^{1 / 2}[(r \partial A / \partial r)(\partial \Omega / \partial z)- \\
& -(r \partial A / \partial z)(\partial \Omega / \partial r)] t+r^{2}[(\partial A / \partial r)(\partial \Omega / \partial z)- \\
& -(\partial A / \partial z)(\partial \Omega / \partial r]^{2} t^{2} .
\end{aligned}
$$

After the function $f(t)$ is reasonably selected, we get the distribution of the angular velocity from Equation (2.12); its characteristic equations are

$$
\frac{\mathrm{d} r}{r(\partial A / \partial z)}=\frac{\mathrm{d} z}{-r(\partial A / \partial r)}=\frac{\mathrm{d} \Omega}{f(t) f^{\prime}(t)\left[f^{2}(t)+G_{1}^{2}(A)\right]^{-1 / 2}} .
$$

The solution of Equation (2.14) gives the distribution of the angular velocity $\Omega$ in the form

$$
\Omega(r, z, t)=H_{1}[t, r, A(r, z)]+H_{2}[A(r, z)],
$$

where the functions $H_{1}$ and $H_{2}$ can be determined by the initial conditions. In Equation (2.15), the first term is introduced by unsteady plasma rotation as a correctional term. Obviously, it is a new term and may reduce to a constant if $f^{\prime}(t)=0$.

In order to give a clear illustration, we discuss briefly an example in which $\alpha_{i}$ is a constant. In this case, Equation (2.10) reduces to the linear force-free equation. Hu (1977) has studied the features of the linear force-free field in detail and obtained its 
general solution in the form

$$
A(r, z)=r \sum_{n=0}^{\infty}\left\{\left[C_{1 n} \exp \left(-\lambda_{n} z\right)+C_{2 n} \exp \left(+\lambda_{n} z\right)\right]\left[C_{3 n} J_{1}\left(\beta_{n} r\right)+C_{4 n} Y_{1}\left(\beta_{n} r\right)\right]\right\},
$$

where $C_{i n}$ can be determined by the boundary value in the axial direction. Both $J_{1}$ and $Y_{1}$ are Bessel functions of the first order. After some reasonable simplification, the magnetic potential can be derived

$$
A(r, z) \cong C_{0} r \exp \left(-\lambda_{0} z\right)\left[J_{1}\left(\beta_{0} r\right)\right]
$$

Substituting Equation (2.16) into (2.15), we get the angular velocity as

$$
\Omega(r, z, t)=\frac{v}{r}=\frac{-r f(t) f^{\prime}(t)(\ln r)}{r \alpha A \sqrt{f^{2}(t)+\alpha^{2} A(r, z)}}+r H_{2}[A(r, z)] .
$$

From Equation (2.8), we can obtain the variational law of the toroidal magnetic energy. In this approximation, its specific expression is

$$
W_{\theta}=B_{\theta}^{2} / 8 \pi=\left(1 / r^{2}\right)\left[f^{2}(t)+C_{0}^{2} r^{2} \exp \left(-2 \lambda_{0} z\right) J_{1}^{2}\left(\beta_{0} r\right)\right] .
$$

Equation (2.18) shows that the magnetic energy including the term of a twisted effect $f(t)$, will be larger than that of the static force-free field. Both Equations (2.8) and (2.18) show that $B_{\theta}(r, z, t)$ has a singularity at $r=0$ if $f(t) \neq 0$. The regular region of the solutions should, therefore, exclude the region near the origin $r=0$.

If the application region includes the region near the origin $r=0$, the regular condition requires $f(t)=0$. In this case, both the poloidal and toroidal magnetic field will be unable to change with time $t$. The continuous configuration of the force-free field in the ideal plasma can, therefore, not be maintained by the pure unsteady plasma rotation. The discussion about the energy storage by a pure azimuthal motion in the solar active region is thus not a perfect approach. We should, furthermore, consider the unsteady poloidal flow field and the unsteady coupling processes between the magnetic field and both the toroidal and the poloidal velocity components.

\section{The Addition of the Unsteady Poloidal Motion}

In the solar active region, the energy source should exist at the lower levels, the photospheric and the convective region. After some conversions and transfer, the energy is stored up at the upper levels, the chromospheric and the coronal region. Svestka (1977), Low (1980), $\mathrm{Li}$ and $\mathrm{Hu}(1982 b, 1984)$ have all come to the same conclusion. Therefore, the poloidal plasma motion should play an important role besides the plasma rotation. An assorted poloidal plasma motion exists in the active region. Athay (1981), Yeh (1981, 1982), Hu (1983a, b), and Li (1985) discussed the features of the poloidal plasma motion. Of course, some reasonable assumption for any practical process have to be made.

Both Equations (1.9) and (1.10) show that when the magnetic field is unsteady, the poloidal velocity field cannot be zero and is also unsteady. The toroidal velocity may 
thus magnify the toroidal magnetic field, and the poloidal velocity may change the poloidal magnetic field. In the same physical problem, both Equations (1.8) and (1.9) should all be linear or all be unlinear in $A$. The linear condition is

$$
G(A, t)[\partial G(A, t) / \partial A]=\alpha_{1}(t) A+\alpha_{2}(t) / 2 .
$$

Solving Equation (3.1), we get

$$
G(A, t)= \pm\left[G_{0}^{2}(t)+\alpha_{1}(t) A^{2}+\alpha_{2}(t) A+\alpha_{3}(t)\right]^{1 / 2} .
$$

Because $G(0, t)=0$, we have $G_{0}^{2}(t)+\alpha_{3}(t)=0$. Therefore, Equation (3.2) may be reduced to

$$
G(A, t)= \pm\left[\alpha_{1}(t) A^{2}+\alpha_{2}(t) A\right]^{1 / 2} .
$$

In this case, the force-free factor may correspondingly be written as

$$
\alpha=\mp\left[\alpha_{1}(t) A+\alpha_{2}(t) / 2\right] /\left[\alpha_{1}(t) A^{2}+\alpha_{2}(t) A\right]^{1 / 2} .
$$

If $\alpha_{2}(t)=0, \alpha$ can be determined from

$$
\alpha^{2}=\alpha_{1}(t) \text {. }
$$

Substituting Equation (3.1) into (1.8), we get the following force-free equation and boundary conditions

$$
\begin{aligned}
& \Lambda(A)+\alpha^{2}(t) A=0, \\
& A(r, 0, t)=f_{1}(r, t), \quad A(r, L, t)=f_{2}(r, t), \\
& A(0, z, t)=0, \quad A(\infty, z, t)=0,
\end{aligned}
$$

where $\alpha$ and $A$ are all a function of time $t$.

If we use the Hankel transformation, we can obtain the solutions of Equations (3.6)-(3.8). In the Hankel transformation, the time $t$ will be used as a parameter. In order to convert Equation (3.6) to a Bessel equation, we have to assume

$$
A_{\mathrm{l}}(r, z, t)=A(r, z, t) / r ;
$$

then, Equations (3.6)-(3.8) become

$$
\begin{aligned}
& \frac{\partial^{2} A_{1}}{\partial r^{2}}+\frac{1}{r} \frac{\partial A_{1}}{\partial r}+\frac{\partial^{2} A_{1}}{\partial z^{2}}+\left(\alpha^{2}-\frac{1}{r^{2}}\right) A_{1}=0, \\
& A_{1}(r, 0, t)=f_{1}(r, t) / r, \quad A_{1}(r, L, t)=f_{2}(r, t) / r, \\
& A_{1}(0, z, t)=0, \quad A_{1}(\infty, z, t)=0 .
\end{aligned}
$$

In terms of a Hankel transform

$$
\widetilde{A}(z, t)=\int_{0}^{\infty} r A_{1}(r, z, t) J_{\mathbf{1}}(\xi, r) \mathrm{d} r
$$


Equations (3.10)-(3.12) may be reduced to

$$
\begin{aligned}
& \left(\partial^{2} \tilde{A} / \partial z^{2}\right)+\left[\alpha^{2}(t)+\xi^{2}\right] \tilde{A}=0, \\
& \tilde{A}(0, t)=F_{1}(\xi, t), \quad \tilde{A}(L, t)=F_{2}(\xi, t),
\end{aligned}
$$

where

$$
F_{i}=\int_{0}^{\infty} f_{i}(r, t) J_{1}(\xi, r) \mathrm{d} r, \quad i=1,2 .
$$

It is not very difficult to determine the solutions of Equations (3.14) and (3.15): namely,

$$
\tilde{A}=c_{1} \exp \left[\left(\alpha^{2}+\xi^{2}\right)^{1 / 2} z\right]+c_{2} \exp \left[-\left(\alpha^{2}+\xi^{2}\right)^{1 / 2} z\right],
$$

where

$$
\begin{aligned}
& c_{1}=\frac{F_{2}(\xi, t)-F_{1}(\xi, t) \exp \left[-\left(\alpha^{2}+\xi^{2}\right)^{1 / 2} L\right]}{2 \sinh \left(\sqrt{\alpha^{2}+\xi^{2}} L\right)}, \\
& c_{2}=\frac{F_{1}(\xi, t) \exp \left[\left(\alpha^{2}+\xi^{2}\right)^{1 / 2} L\right]-F_{2}(\xi, t)}{2 \sinh \left(\sqrt{\alpha^{2}+\xi^{2}} L\right)} .
\end{aligned}
$$

An inversion of the Hankel transform leads to

$$
\begin{aligned}
A_{1}(r, z, t)= & \int_{0}^{\infty}\left\{F_{1}(\xi, t) \frac{\sinh \left[\sqrt{\alpha^{2}+\xi^{2}}(L-z)\right]}{\sinh \left(\sqrt{\alpha^{2}+\xi^{2}} L\right)}+\right. \\
& \left.+\frac{\sinh \left(\sqrt{\alpha^{2}+\xi^{2}}+z\right)}{\sinh \left(\sqrt{\alpha^{2}+\xi^{2}} L\right)} F_{2}(\xi, t)\right\} \xi J_{1}(\xi, r) \mathrm{d} \xi .
\end{aligned}
$$

Because the solution of Equations (3.6)-(3.8) is

$$
A(r, z, t)=r A_{1}(r, z, t) \text {. }
$$

Substituting Equation (3.21) into (3.3) and according to Equation (1.7), we can get $G(r, z, t)$ and $B_{\theta}(r, z, t)$. We can now discuss the evolution of the toroidal magnetic field with time $t$. For example, we analyze brief by a condition in which $\alpha_{2}(t)=0$. In this case, we have

$$
\begin{aligned}
G(r, z, t)= & r \propto\left\{\int _ { 0 } ^ { \infty } \left[F_{1}(\xi, t) \frac{\sinh \left[\sqrt{\alpha^{2}+\xi^{2}}(L-z)\right]}{\sinh \left(\sqrt{\alpha^{2}+\xi^{2}} L\right)}+\right.\right. \\
& \left.\left.+\frac{\sinh \left(\sqrt{\alpha^{2}+\xi^{2}} z\right)}{\sinh \left(\sqrt{\alpha^{2}+\xi^{2}} L\right)} F_{2}(\xi, t)\right] \xi J_{1}(\xi, r) \mathrm{d} \xi\right\} .
\end{aligned}
$$


The density of the toroidal magnetic energy is then

$$
W_{\theta}=B_{\theta}^{2} / 8 \pi=G^{2}(A, t) / 8 \pi r^{2} .
$$

From Equations (3.22) and (3.23), we know that the toroidal magnetic energy exists when the force-free factor $\alpha$ increases with time $t$. Due to the addition of the poloidal plasma motion, an unsteady process which causes the change of $\alpha$ with time can be maintained in the active region. The change of $\alpha$ with time mirrors some effects of the plasma motion in the solar atmosphere.

We can now derive a kinematical form. Due to the poloidal plasma motion, the kinetic energy and the internal energy of the plasma under the lower levels may be transferred to the upper levels of the active region. The coupling process between the magnetic field and the flow field will cause the enhancement of the force-free factor $\alpha$ with time $t$. The enhancing $\alpha$ will impel the increase in the toroidal magnetic field. If also plasma rotation exists at the same time, the toroidal magnetic field can increase even more. In this case, the energy may be stored up in the active region. Due to the continued enhancement of $W_{\theta}$, it is possible that the active region becomes unstable and an eruption occurs.

\section{A Storage Mechanism Caused by the Poloidal Flow}

When we have poloidal plasma motion in the active region, the plasma rotation may be either existent or inexistent. In fact, even if there is no twisted magnetic line of force in the active region, the energy of the force-free magnetic field can still be increased and stored. In order to expound this point clearly, we discuss an example in which the energy is continuously transferred by the poloidal flow. We assume a flow field as

$$
u=0, \quad w=w(t) .
$$

According to the frozen condition (1.9), we have

$$
\partial A / \partial t+w(t)(\partial A / \partial z)=0 .
$$

Its characteristic equation is

$$
\mathrm{d} t / 1=\mathrm{d} z / w(t) .
$$

From Equation (4.3), we have

$$
z-s(t)=c,
$$

where $c$ is a constant, and

$$
s(t)=\int_{0}^{t} w(t) \mathrm{d} t .
$$

Thus, the magnetic potential $A$ which can be obtained from Equation (4.2) can be expressed by

$$
A=F[r, z-s(t)],
$$


where $F$ is an unknown function. In addition, we assume that $G(A, t)$, a double function of $A$ and $t$, satisfies

$$
\partial G(A, t) / \partial t=0
$$

In this case, the change of $G(A, t)$ is caused by the change of $A(r, z, t)$ with time. Using Equations (4.1) and (4.7), the frozen condition can be reduced to

$$
w=w(A, t),
$$

where the angular velocity is changing with time $t$ on every magnetic plane.

Now we discuss the force-free field equation (1.8). If we discuss the linear force-free field, Equation (1.8) becomes (3.6). With the condition (4.6), we analyze such a solution which can be expressed in the following form

$$
A=N_{0} r J_{1}(a r) \exp \{-b[z-s(t)]\},
$$

where $N_{0}, a$, and $b$ are some suitable constants. Then, it is easy to verify Equation (3.6) as

$$
\Lambda(A)=\left(b^{2}-a^{2}\right) A
$$

Substituting Equation (4.10) into (1.7), we get

$$
\begin{aligned}
& G(A)=\sqrt{\left(a^{2}-b^{2}\right)} A(r, z, t), \\
& \alpha=\sqrt{\left(a^{2}-b^{2}\right)}=\text { constant } .
\end{aligned}
$$

According to Equations (4.9) and (4.11), we obtain the contribution of the magnetic field as follows

$$
\begin{aligned}
& B_{r}=N_{1} J_{1}(a r) \exp \{-b[z-s(t)]\}, \\
& B_{\theta}=N_{2} J_{1}(a r) \exp \{-b[z-s(t)]\}, \\
& B_{z}=N_{3} J_{0}(a r) \exp \{-b[z-s(t)]\} .
\end{aligned}
$$

From Equations (4.13), (4.14), and (4.15), we get the magnetic energy of the active region; it is

$$
W=M \exp [2 b s(t)][1-\exp (-2 b L)]\left[J_{1}^{2}(a R)+J_{0}^{2}(a R)\right],
$$

where $N_{1}=-N_{0}, N_{2}=-N_{0}\left(a^{2}-b^{2}\right)^{1 / 2}, N_{3}=-a N_{0}, M=N_{0} a^{2} R^{2} / 16 b$ are some suitable constants which can all be determined. The limits of the active region is taken as $r \leq R, 0 \leq z \leq L$.

Equations (4.13)-(4.15) show that the magnetic field changes with time, and Equation (4.16) shows that the magnetic energy does the same. From Equation (4.16) we know that the energy in the force-free field region may either be stored up or be dissipated. Observations have shown that the magnetic field intensity gradually decreases with the enhancement of $z$. Thus, the constant which is in the exponential term of Equation (4.9) must be positive, i.e., $b>0$. Whether the magnetic energy increases 
or decreases will, therefore, be determined by the algebraic symbol of $s(t)$. When $s(t)>0$, the magnetic energy $W$ increases with time $t$ and the direction of the average motion is up. When $s(t)<0$, then $W$ decreases with $t$, i.e., the axial average motion is down. The behaviour of $s(t)$ is determined by the environment.

Since the magnetic energy increases when the plasma goes up, the energy of the lower levels is transferred to the upper levels by the plasma motion. We have an assorted axial motion in the solar atmosphere. It should eventually be possible for the "up motion' to dominate over the 'down motion' for some time. In this case, considerable energy can be stored in the upper levels of the active region.

We can now make a quantitative estimate. We suppose that the energy of the active region has already increased $10 \%$ during one day. From Equation (4.16), we have

$$
(W) t /(W)_{0}=\exp \left[2 b \int_{0}^{t} w(t) \mathrm{d} t\right]=11 / 10, \quad t=24 \text { hours } .
$$

The order of magnitude of $b$ should be proportional to $1 / R$, with the characteristic size $R \cong 4 \times 10^{9} \mathrm{~cm}$. The order of magnitude of the 'up motion' will then be

$$
w=20 \mathrm{~m} \mathrm{~s}^{-1} \text {. }
$$

If such an axial plasma motion is continuously maintained and kept at $20 \mathrm{~m} \mathrm{~s}^{-1}$ for one day, then a sizeable fraction of the energy may be stored in the active region. It is sufficient for the eruption of a solar flare. Due to the features of the poloidal motion in the solar atmosphere, it is possible that the 'up motion' dominates.

When the force-free factor $\alpha$ increases, the magnetic lines of force are twisted. It might be a general storage process of the energy in the active region, but others exist as well. As long as the energy under the lower levels can be transferred to the upper levels, the energy can be stored as well even if there is no twisted line of force. There should be several storage processes in the active region. But the storage of the energy of the force-free field should all be caused by an outside energy flow.

Since there are several processes, the problem is not very simple. The above discussion might help to understand the energy conversions in the force-free frozen magnetic field, as well as many associated phenomena in the active region.

\section{Acknowledgements}

One of the authors (L.Z.) would like to thank Prof. J. Rahe and Dr H. Drechsel for their hospitality during his stay visit at the Remeis Observatory in Bamberg. He would also like to thank the Max-Planck-Gesellschaft for the fellowship support.

\section{References}

Anzer, U.: 1968, Solar Phys. 3, 298.

Anzer, U. and Pneuman, G. W.: 1982, Solar Phys. 79, 129

Athay, G.: 1981, in Q. Orall (ed.), Observation of Mass Motion in Active Region, p. 83. 
Barnes, C. W. and Sturrock, P. A.: 1972, Astrophys. J. 174, 659.

Bostrom, R.: 1973, Astrophys. Space Sci. 22, 353.

Gold, T. and Hoyle, F.: 1960, Monthly Notices Roy. Astron. Soc. 120, 89.

Hu, W. R.: 1977, Scientia Sinica 1, 69.

Hu, W. R.: 1983a, Solar Phys. 83, 195.

Hu, W. R.: 1983b, Astrophys. Space Sci. 90, 391.

Hu, W. R.: 1983c, Astrophys. Space Sci. 92, 395.

Li, Z.: 1985, Acta Astrophys. Sinica 5, 1.

$\mathrm{Li}, Z .: 1986, \operatorname{ICTP} / 85 / 158$.

Li, Z. and Hu, W. R.: 1982a, J. USTC Suppl. 1, 137.

Li, Z. and Hu, W. R.: 1982b, Chinese J. Space Sci. 2, 137.

Li, Z. and Hu, W. R.: 1984, Mon. J. Sci. 29, 980.

Low, B. C.: 1980, Solar Phys. 67, 57.

Ma, Y.: 1981, The Proceedings of Huangshan Conference on the Astrophysics, Sci. Press, p. 202.

Švestka, Z.: 1977, Solar Flares, D. Reidel Publ. Co., Dordrecht, Holland, pp. 300-310.

Yeh, T.: 1981, Astrophys. J. 254, 704.

Yeh, T.: 1982, Solar Phys. 78, 287. 